\title{
Total Eccentricity Index of the Generalized Hierarchical Product of Graphs
}

\author{
Nilanjan De · Sk. Md. Abu Nayeem • Anita Pal
}

Published online: 9 November 2014

(C) Springer India Pvt. Ltd. 2014

\begin{abstract}
Let $G=(V(G), E(G))$ be a connected graph. The total eccentricity index of $G$ is defined as $\zeta(G)=\sum_{v \in V(G)} \varepsilon_{G}(v)$, where $\varepsilon_{G}(v)$ is the eccentricity of the vertex $v$. In this paper, we compute the total eccentricity of generalized hierarchical product of graphs. Moreover, we derive some explicit formulae for total eccentricity index of F-sum graph, Cartesian product, Cluster product and Corona product of graphs and apply those results to find the total eccentricity index of various classes of chemical graphs and nanostructures.
\end{abstract}

Keywords Eccentricity · Graph invariant · Total eccentricity index · Graph operations

Mathematics Subject Classification Primary: 05C35 - Secondary: 05C07, 05C40

\section{Introduction}

Let $G$ be a connected graph with vertex set $V(G)$ and edge set $E(G)$. As usual, the cardinality of $V(G)$ and $E(G)$ are denoted by $|V(G)|$ and $|E(G)|$, respectively. For any two vertices $u, v \in V(G)$, the distance between $u$ and $v$ is denoted by $d_{G}(u, v)$ and is given by the number of edges in the shortest path connecting $u$ and $v$. Also we denote the sum of distances between $v \in V(G)$ and every other vertices in $G$ by $d(x \mid G)$, i.e., $d(x \mid G)=\sum_{v \in V(G)} d_{G}(x, v)$. The

\section{N. De $(\bowtie)$}

Department of Basic Sciences and Humanities (Mathematics), Calcutta Institute of Engineering and Management, Kolkata, India

e-mail: de.nilanjan@rediffmail.com

S. M. Abu Nayeem

Department of Mathematics, Aliah University, DN - 20, Sector - V, Salt Lake,

Kolkata 700 091, India

e-mail: nayeem.math@aliah.ac.in

A. Pal

Department of Mathematics, National Institute of Technology, Durgapur, India e-mail: anita.buie@gmail.com 
eccentricity of a vertex $v$, denoted by $\varepsilon_{G}(v)$, is the largest distance from $v$ to any other vertex $u$ of $G$. Different topological indices based on vertex eccentricity are already subject to various mathematical and chemical study. The total eccentricity index of $G$ is defined as $\zeta(G)=\sum_{v \in V(G)} \varepsilon_{G}(v)$. Fathalikhani et al. [12] have studied total eccentricity of some graph operations. Some studies on average eccentricities are also found in literature [5,17].

If two graphs $G$ and $H$ are isomorphic, we write $G \cong H$. Let $T o p(G)$ represents certain topological index of $G$ and if $G \cong H$, then $\operatorname{Top}(G)=\operatorname{Top}(H)$. Let $L(G)$ denotes the line graph of $G$, is the graph whose vertices are the edges of $G$ and two vertices of $L(G)$ are adjacent if and only if the corresponding edges are adjacent in $G$.

As an extension of Cartesian product graphs, Barrière et al. $[3,4]$ introduced the generalized hierarchical product of graphs. Many results on different topological indices of generalized hierarchical product of graphs are reported in the literature $[1,2,10,15]$. Eliasi and Taeri [9] introduced the F-sum graphs. It is evident that the Cartesian product, F-sum product and cluster product are special cases of generalized hierarchical product and thus the results for these products can be deduced from those of generalized hierarchical product of graphs.

In this paper, the total eccentricity index of the generalized hierarchical product of graphs is computed. Also we find the total eccentricity indices of Cartesian product graph, the Fsum graph, the cluster product graph and the corona product graph as some special cases. In addition to these, as applications, we present explicit results for total eccentricity index of different classes of chemical graphs and nanostructurs such as $C_{4}$-nanotubes, $C_{4}$-nanotorus, zig zag polyhex nanotube, the hexagonal chain and so on.

\section{Generalized Hierarchical Product of Graphs}

Definition 1 Let $G$ and $H$ be two connected graphs and $U$ be a non-empty subset of $V(G)$. Then the hierarchical product of $G$ and $H$, denoted by $G(U) \Pi H$, is the graph with vertex set $V(G) \times V(H)$, and any two vertices $(u, v)$ and $\left(u^{\prime}, v^{\prime}\right)$ of $G(U) \Pi H$ are adjacent if and only if $\left[u=u^{\prime} \in V(G)\right.$ and $\left.\left(v, v^{\prime}\right) \in E(H)\right]$ or $\left[v=v^{\prime} \in V(H)\right.$ and $\left.\left(u, u^{\prime}\right) \in E(G)\right]$.

A path between the vertices $u, v \in V(G)$ through $U \subseteq V(G)$ is a $u v$-path in $G$ containing some vertex $x \in U$ (vertex $x$ could be the vertex $u$ or $v$ ). The distance between $u$ and $v$ through $U$ is the length of the shortest path between $u$ and $v$ through $U$ and is denoted by $d_{G(U)}(u, v)$. If $u \in U$, then we have,

$$
\varepsilon_{G(U)}(u)=\max _{u \in V(G)} d_{G(U)}(u, v) \text { and } \zeta(G(U))=\sum_{u_{i} \in V(G)} \varepsilon_{G(U)}\left(u_{i}\right) .
$$

Lemma 1 [4] Let $G$ and $H$ be two connected graphs and $U \subseteq V(G)$. Then $\varepsilon_{G(U) \Pi H}(u, v)$ $=\varepsilon_{G(U)}(u)+\varepsilon_{H}(v)$.

Theorem 1 Let $G$ and $H$ be two connected graphs and $U \subseteq V(G)$. Then

$$
\zeta(G(U) \Pi H)=|V(H)| \zeta(G(U))+|V(G)| \zeta(H) .
$$

Proof Let $V(G)=\left\{u_{1}, u_{2}, \ldots, u_{n}\right\}$ and $V(H)=\left\{v_{1}, v_{2}, \ldots, v_{m}\right\}$. Then, using definition of total eccentricity index and Lemma 1 , we have 


$$
\begin{aligned}
\zeta(G(U) \Pi H) & =\sum_{\left(u_{i}, v_{j}\right)} \varepsilon_{G(U) \Pi H}\left(u_{i}, v_{j}\right) \\
& =\sum_{u_{i} \in V(G)} \sum_{v_{j} \in V(H)}\left\{\varepsilon_{G(U)}\left(u_{i}\right)+\varepsilon_{H}\left(v_{j}\right)\right\} \\
& =|V(H)| \sum_{u_{i} \in V(G)} \varepsilon_{G(U)}\left(u_{i}\right)+|V(G)| \sum_{v_{j} \in V(H)} \varepsilon_{H}\left(v_{j}\right) \\
& =|V(H)| \zeta(G(U))+|V(G)| \zeta(H) .
\end{aligned}
$$

This completes the proof.

Definition 2 Let $G$ and $H$ be two connected graphs. The Cartesian product of $G$ and $H$, denoted by $G \square H$, is the graph with vertex set $V(G) \times V(H)$, and any two vertices $\left(u_{p}, v_{r}\right)$ and $\left(u_{q}, v_{s}\right)$ are adjacent if and only if $\left[u_{p}=u_{q} \in V(G)\right.$ and $\left.\left(v_{r}, v_{s}\right) \in E(H)\right]$ or $\left[v_{r}=v_{s} \in V(H)\right.$ and $\left.\left(u_{p}, u_{q}\right) \in E(G)\right]$ and $r, s=1,2, \ldots,|V(H)|$.

From definition of the Cartesian product graph it is clear that, the Cartesian product of graphs is a special case of generalized hierarchical product graph, that is, if $U=V(G)$, then $G(U) \Pi H \cong G \square H$. So, from the previous theorem the following corollary follows.

Corollary 1 Let $G$ and $H$ be two connected graphs, then

$$
\zeta(G \square H)=|V(H)| \zeta(G)+|V(G)| \zeta(H) .
$$

Fathalikhani et al. [12] obtained the same result from direct calculation.

Using (2), now we compute total eccentricity of the $C_{4}$-nanotorus, nanotube and also for polynomial chain.

Let $P_{n}(n \geq 1)$ and $C_{n}(n \geq 3)$ be path and cycle of order $n$ respectively, then we have

$$
\zeta\left(P_{n}\right)= \begin{cases}\frac{3}{4} n^{2}-\frac{1}{2} n, & \text { when } n \text { is even } \\ \frac{3}{4} n^{2}-\frac{1}{2} n-\frac{1}{4}, & \text { when } n \text { is odd, }\end{cases}
$$

and

$$
\zeta\left(C_{n}\right)= \begin{cases}\frac{1}{2} n^{2}, & \text { when } n \text { is even } \\ \frac{1}{2} n(n-1), & \text { when } n \text { is odd. }\end{cases}
$$

Example 1 The $C_{4}$-nanotorus can be viewed as the Cartesian product of $C_{m}$ and $C_{n}$, i.e., $T C_{4}(m, n)=C_{m} \square C_{n}$. So, its total eccentricity is given by

$\zeta\left(T C_{4}(m, n)\right)= \begin{cases}\frac{1}{2} m n(m+n), & \text { when } m, n \text { both are even, } \\ \frac{1}{2} m n(m+n-2), & \text { when } m, n \text { both are odd } \\ \frac{1}{2} m n(m+n-1), & \text { when either of } m, n \text { is odd and the other is even. }\end{cases}$

Example 2 The $C_{4}$-nanotube can be viewed as the Cartesian product of $P_{n}$ and $C_{m}$, i.e., $T U C_{4}(m, n)=P_{n} \square C_{m}$. So, its total eccentricity is given by

$$
\zeta\left(T U C_{4}(m, n)\right)= \begin{cases}\frac{1}{2} m n\left(\frac{3}{2} m+n-1\right), & \text { when } m, n \text { both are even, } \\ m n\left(\frac{3}{4} m+\frac{1}{2} n-1\right)-\frac{1}{4} n, & \text { when } m, n \text { both are odd, } \\ \frac{1}{2} m n\left(\frac{3}{2} m+n-2\right), & \text { when } m \text { is even and } n \text { is odd, } \\ \frac{1}{2} m n\left(\frac{3}{2} m+n-1\right)-\frac{1}{4} n, & \text { when } m \text { is odd and } n \text { is even. }\end{cases}
$$


Example 3 The $m \times n$ grid graph can be viewed as the Cartesian product of $P_{n}$ and $P_{m}$, i.e., $G_{m, n}=P_{n} \square P_{m}$. So, the total eccentricity of the grid graph $G_{m, n}$ is given by

$$
\zeta\left(G_{m, n}\right)= \begin{cases}\frac{3}{4} m n(m+n)-m n, & \text { when } m, n \text { both are even, } \\ \frac{1}{4} m n(m+n)-m n, & \text { when } m, n \text { both are odd, } \\ \frac{1}{4} m n(m+n)-m n-\frac{1}{4} m, & \text { when } m \text { is even and } n \text { is odd, } \\ \frac{3}{4} m n(m+n)-m n-\frac{1}{4} n, & \text { when } m \text { is odd and } n \text { is even. }\end{cases}
$$

\section{F-sum Graphs}

The concept of F-sum graph was first introduced by Eliasi and Taeri [9] and the Weiner indices of the resulting graphs were studied therein. Li and Wang [14] derived explicit expression of the PI indices of four sums of two graphs. The hyper and reverse Weiner indices of Fsum graphs were studied by Metsidik et al. [16]. Eskender and Vumar [11] determined the eccentric connectivity index of F-sum graphs in terms of some invariants of the factors.

The subdivision graph of a graph $G$, denoted by $S(G)$, is obtained from $G$ by replacing each edge of $G$ by a path of length two. The triangle parallel graph of a graph $G$ is denoted by $R(G)$ and is obtained from $G$ by replacing each edge of $G$ by a triangle. The line superposition graph $Q(G)$ of a graph $G$ is obtained from $G$ by inserting a new vertex into each edge of $G$ and then joining with edges each pair of new vertices on adjacent edges of $G$. The total graph $T(G)$ of a graph $G$ has its vertices as the edges and vertices of $G$ and adjacency in $T(G)$ is defined by the adjacency or incidence of the corresponding elements of $G$.

Definition 3 Let $\mathrm{F}$ be one of the subdivision operations $S, Q, R$ or $T$. For two connected graphs $G$ and $H$, the F-sum, denoted by $G+_{F} H$, is the graph with vertex set $(V(G) \cup$ $E(G)) \times V(H)$, and any two vertices $(u, v)$ and $\left(u^{\prime}, v^{\prime}\right)$ of $G+{ }_{F} H$ are adjacent if and only if $\left[u=u^{\prime} \in V(G)\right.$ and $\left.\left(v, v^{\prime}\right) \in E(H)\right]$ or $\left[v=v^{\prime} \in V(H)\right.$ and $\left.\left(u, u^{\prime}\right) \in E(F(G))\right]$.

Lemma 2 [11] Let $G$ and $H$ be two connected graphs. If $U=V(G)$, then we have

a. $|V(S(G))|=|V(G)|+|E(G)|,|E(S(G))|=2|E(G)|$.

b. For each vertex $v \in U, \varepsilon_{S(G)(U)}(v)=2 \varepsilon_{G}(v)$.

c. For each vertex $v \in V(S(G)) \backslash U, \varepsilon_{S(G)(U)}(v)=2 \varepsilon_{L(G)}(v)+1$.

Theorem 2 Let $G(n \geq 2)$ and $H$ be two connected graphs. Then,

$$
\zeta\left(G+{ }_{S} H\right)=|V(H)|[2 \zeta(G)+\zeta(L(G))+\mid E(G)]+[|V(G)|+|E(G)|] \zeta(H) .
$$

Proof If $U$ be a non-empty subset of $V(S(G))$, we have by Lemma 2,

$$
\begin{aligned}
\zeta(S(G)(U)) & =\sum_{v \in V(S(G))} \varepsilon_{S(G)(U)}(v) \\
& =\sum_{v \in U} \varepsilon_{S(G)(U)}(v)+\sum_{v \in V(S(G)) \backslash U} \varepsilon_{S(G)(U)}(v) \\
& =2 \sum_{v \in U} \varepsilon_{G}(v)+\sum_{v \in V(L(G))}\left(2 \varepsilon_{L(G)}(v)+1\right) \\
& =2 \zeta(G)+\zeta(L(G))+|E(G)| .
\end{aligned}
$$

Combining this result with (1) we get the desired result. 
Lemma 3 [11] Let $G$ and $H$ be two connected graphs. If $U=V(G)$, then we have

a. $|V(R(G))|=|V(G)|+|E(G)|,|E(R(G))|=3|E(G)|$.

b. For each vertex, $v \in U, \varepsilon_{R(G)(U)}(v)=\varepsilon_{G}(v)$.

c. For each vertex, $v \in V(R(G)) \backslash U, \varepsilon_{R(G)(U)}(v)=\varepsilon_{L(G)}(v)+1$.

Theorem 3 Let $G(n \geq 2)$ and $H$ be two connected graphs. Then

$$
\zeta\left(G+{ }_{R} H\right)=|V(H)|[\zeta(G)+\zeta(L(G))+\mid E(G)]+[|V(G)|+|E(G)|] \zeta(H) .
$$

Proof If $U$ be a non-empty subset of $V(R(G))$, we have using the Lemma 3,

$$
\begin{aligned}
\zeta(R(G)(U)) & =\sum_{v \in V(R(G))} \varepsilon_{R(G)(U)}(v) \\
& =\sum_{v \in U} \varepsilon_{R(G)(U)}(v)+\sum_{v \in V(R(G)) \backslash U} \varepsilon_{R(G)(U)}(v) \\
& =2 \sum_{v \in U} \varepsilon_{G}(v)+\sum_{v \in V(L(G))}\left(\varepsilon_{L(G)}(v)+1\right) \\
& =\zeta(G)+\zeta(L(G))+|E(G)| .
\end{aligned}
$$

Again using (1) we get the desired result.

Lemma 4 [11] Let $G$ and $H$ be two connected graphs. If $U=V(G)$, then we have

a. $|V(Q(G))|=|V(G)|+|E(G)|,|E(Q(G))|=2|E(G)|+|E(L(G))|$.

b. For each vertex, $v \in U, \varepsilon_{Q(G)(U)}(v)=\varepsilon_{G}(v)+1$.

c. For each vertex, $v \in V(Q(G)) \backslash U, \varepsilon_{Q(G)(U)}(v)=\varepsilon_{L(G)}(v)+1$.

Theorem 4 Let $G(n \geq 2)$ and $H$ be two connected graphs. Then

$\zeta\left(G+{ }_{Q} H\right)=|V(H)|[\zeta(G)+\zeta(L(G))+|V(G)|+\mid E(G)]+[|V(G)|+|E(G)|] \zeta(H)$.

Proof We have using the Lemma 4, if $U$ be a non-empty subset of $V(R(G))$,

$$
\begin{aligned}
\zeta(Q(G)(U)) & =\sum_{v \in V(Q(G))} \varepsilon_{Q(G)(U)}(v) \\
& =\sum_{v \in U} \varepsilon_{Q(G)(U)}(v)+\sum_{v \in V(Q(G)) \backslash U} \varepsilon_{Q(G)(U)}(v) \\
& =2 \sum_{v \in U}\left(\varepsilon_{G}(v)+1\right)+\sum_{v \in V(L(G))}\left(\varepsilon_{L(G)}(v)+1\right) \\
& =\zeta(G)+|V(G)|+\zeta(L(G))+|E(G)| .
\end{aligned}
$$

As before, we get the desired result from (1).

Lemma 5 [11] Let $G$ and $H$ be two connected graphs. If $U=V(G)$, then we have

a. $|V(T(G))|=|V(G)|+|E(G)|,|E(T(G))|=3|E(G)|+|E(L(G))|$.

b. For each vertex, $v \in U, \varepsilon_{T(G)(U)}(v)=\varepsilon_{G}(v)$.

c. For each vertex, $v \in V(T(G)) \backslash U, \varepsilon_{T(G)(U)}(v)=\varepsilon_{L(G)}(v)+1$.

Theorem 5 Let $G(n \geq 2)$ and $H$ be two connected graphs. Then

$$
\zeta\left(G+{ }_{T} H\right)=|V(H)|[\zeta(G)+\zeta(L(G))+\mid E(G)]+[|V(G)|+|E(G)|] \zeta(H) .
$$


Proof We have using the last Lemma 5, if $U$ be a non-empty subset of $V(R(G))$,

$$
\begin{aligned}
\zeta(T(G)(U)) & =\sum_{v \in V(T(G))} \varepsilon_{T(G)(U)}(v) \\
& =\sum_{v \in U} \varepsilon_{T(G)(U)}(v)+\sum_{v \in V(T(G)) \backslash U} \varepsilon_{T(G)(U)}(v) \\
& =\sum_{v \in U} \varepsilon_{G}(v)+\sum_{v \in V(L(G))}\left(\varepsilon_{L(G)}(v)+1\right) \\
& =\zeta(G)+\zeta(L(G))+|E(G)| .
\end{aligned}
$$

Thus, we get the desired result from (1).

Let $U=V\left(C_{n}\right)$, where $C_{n}(n \geq 3)$ be a cycle, then from (3), we have the following corollary.

Corollary 2 Let $H$ be an arbitrary connected graph, then

$$
\zeta\left(C_{n}+{ }_{S} H\right)= \begin{cases}2 n \zeta(H)+n(2 n+1)|V(H)|, & \text { when } n \text { is even } \\ 2 n \zeta(H)+n(2 n-1)|V(H)|, & \text { when } n \text { is odd } .\end{cases}
$$

Example 4 Let $L_{n} \cong P_{n+1}+{ }_{S} P_{2}$ be a linear hexagonal chain [8] with $n \geq 2$. Then using (3), we have $\zeta\left(L_{n}\right)=6 n^{2}+8 n+2$.

Example 5 Let $\Gamma$ be the zigzag polyhex nanotube $T U H C 6[2 n, 2]$ with $n \geq 3$. Since $\Gamma \cong$ $C_{n}+{ }_{S} P_{2}$, using (3), we have

$$
\zeta(\Gamma)= \begin{cases}4 n^{2}+6 n, & \text { when } n \text { is even } \\ 4 n(n-1)+6 n, & \text { when } n \text { is odd. }\end{cases}
$$

\section{Total Eccentricity Index of Cluster and Corona Product of Graphs}

Definition 4 The cluster product of two graph $G$ and $H$, denoted by $G\{H\}$, is obtained by taking one copy of $G$ and $|V(G)|$ copies of a rooted graph $H$ and by identifying the root of the $i$-th copy of $H$ with the $i$-th vertex of $G$ for $i=1,2, \ldots,|V(G)|$.

If $|V(G)|=n,|E(G)|=q$ and $|V(H)|=n^{\prime},|E(H)|=q^{\prime}$, then from definition of cluster product graphs $|V(G\{H\})|=n n^{\prime}$ and $|E(G\{H\})|=\left(q+n q^{\prime}\right)$. Let the root vertex of $H$ is denoted by $x$. Note that, if $U=\{x\} \subset V(G)$ then $G\{H\} \cong H(U) \Pi G \cong H(\{x\}) \Pi G$. Then from (1), we have the following results.

Theorem 6 Let $G$ and $H$ be two connected graphs and $x$ is the root vertex of $H$, then

$$
\zeta(G\{H\})=|V(G)|\left[|V(H)| \varepsilon_{H}(x)+d(x \mid H)\right]+\mid \zeta(G)
$$

Proof Since, $U=\{x\} \subset V(H)$, so

$$
\zeta(H(U))=\zeta(H(\{x\}))=|V(H)| \varepsilon_{H}(x)+d(x \mid H) .
$$

So, using equation (1) we get the desired result. 
For a given graph $G$, its $t$-thorny graph $G^{t}$ is obtained by attaching $t$ thorns (degree 1 vertices) to each vertex of $G$. This type of graph was introduced by Gutman [13] and some eccentricity related thorny graphs are studied by De [6,7]. On the other hand, we can also obtain the $t$-thorny graph $G^{t}$ by cluster product of $G$ and the star graph $S_{t+1}$ with $(t+1)$ vertices, where the root of $S_{t+1}$ is on its vertex of degree $t$. This type of cluster product graph is known as $t$-fold bristled graph $\operatorname{Brst}(G)$. So by the previous theorem the total eccentricity index of $t$-thorny graph $G^{t}$ or $t$-fold bristled graph $\operatorname{Brst}(G)$ can easily be computed.

Corollary 3 The total eccentricity index of the t-thorn graph $G^{t}$ is computed as follows

$$
\zeta\left(G^{t}\right)=(t+1) \zeta(G)+n(2 t+1) .
$$

Proof Let $H \cong S_{t+1}$, with root of $S_{t+1}$ being the central vertex of the star which is of degree $t$. Then, we have $\varepsilon_{H}(x)=1, d(x \mid(H)=t$ and $|V(H)|=(t+1)$.

So, using last theorem the desired result follows.

From the above corollary, the total eccentricity index of the $t$-fold bristled graph of $C_{n}$ and $P_{n}$ can easily be computed.

Example 6 The total eccentricity index of $t$-thorny ring $C_{n}^{t}$ is given by

$$
\zeta\left(C_{n}^{t}\right)= \begin{cases}\frac{1}{2} n^{2} t+2 n t+\frac{1}{2} n^{2}+n, & \text { when } n \text { is even } \\ \frac{1}{2} n^{2} t+\frac{3}{2} n t+\frac{1}{2} n^{2}+\frac{1}{2} n, & \text { when } n \text { is odd. }\end{cases}
$$

Example 7 The total eccentricity index of $t$-thorny ring $P_{n}^{t}$ is given by

$$
\zeta\left(P_{n}^{t}\right)= \begin{cases}\frac{3}{4} n^{2} t+\frac{3}{2} n t+\frac{3}{4} n^{2}+\frac{1}{2} n, & \text { when } n \text { is even } \\ \frac{3}{4} n^{2} t+\frac{3}{2} n t+\frac{3}{4} n^{2}+\frac{1}{2} n-\frac{1}{4}(t+1), & \text { when } n \text { is odd. }\end{cases}
$$

Example 8 The square comb lattice $C q(N)$ is the cluster product graph $P_{n}\left\{P_{n}\right\}$, with $N=n^{2}$ number of vertices and the root of $P_{n}$ is on the vertex of degree one. So using (6), its total eccentricity index is given by

$$
\zeta(C q(N))= \begin{cases}n^{2}\left(\frac{9}{4} n-2\right), & \text { when } n \text { is even } \\ n^{2}\left(\frac{9}{4} n-2\right)-\frac{1}{4} n, & \text { when } n \text { is odd. }\end{cases}
$$

Now let us compute the total eccentricity index of the corona product of graphs.

Definition 5 The corona product of two graphs $G$ and $H$ denoted by $G \odot H$, is a graph obtained by taking one copy of $G$ and $|V(G)|$ copies of $H$ and joining the i-th vertex of $G$ to every vertex of in the copy of $H$, for $i=1,2, \ldots,|V(G)|$. According to definition of corona product graph $|V(G \odot H)|=\left(n n^{\prime}+n\right)$ and $|E(G \odot H)|=\left(q+n q^{\prime}+n n^{\prime}\right)$.

Corollary 4 Let $G$ be a connected $(n, q)$ graph and $H$ is $\left(n^{\prime}, q^{\prime}\right)$ graph. Then

$$
\zeta(G \odot H)=n\left(2 n^{\prime}+1\right)+\left(n^{\prime}+1\right) \zeta(G)
$$

Proof For any graph $H$, let $|V(H)|=n$ and $|E(H)|=q$. Also let $x$ be the root vertex of the join of the graphs $H$ and $K_{1}$, denoted by $(H+x)$. Then graph $|V(H+x)|=n^{\prime}+1$ and $|E(H)|=n^{\prime}+q^{\prime}$. Also note that,

$$
\varepsilon_{(H+x)}(x)=1, d(x \mid(H+x))=n^{\prime} \text { and } G \odot H=G(\{H+x\}) .
$$


So from the relation (6), we have

$$
\begin{aligned}
\zeta(G\{H+x\}) & =|V(G)|\left[|V(H+x)| \varepsilon_{H+x}(x)+d(x \mid H+x)\right]+|V(H+x)| \zeta(G) \\
& =n\left[\left(n^{\prime}+1\right)+n^{\prime}\right]+\left(n^{\prime}+1\right) \zeta(G) .
\end{aligned}
$$

as desired.

Remark 1 Note that, for a given graph $G, G\left\{S_{t+1}\right\}=G \odot \bar{K}_{t}$, where $K_{t}$ is the complete graph with $t$ vertices and $S_{t+1}$ is the star graph on $(t+1)$ vertices, with the root of $S_{t+1}$ as the central vertex which is of degree $t$. Here we can easily verify that

$$
\zeta\left(G\left\{S_{t+1}\right\}\right)=\zeta\left(G \odot \bar{K}_{t}\right)=n(2 t+1)+(t+1) \zeta(G) .
$$

Example 9 Let $H$ be any graph with $n$ vertices, then using (11) the following result follows.

$$
\zeta\left(P_{m} \odot H\right)= \begin{cases}m(2 n+1)+(n+1)\left(\frac{3}{4} m^{2}-\frac{1}{2} m\right), & \text { when } m \text { is even } \\ m(2 n+1)+(n+1)\left(\frac{3}{4} m^{2}-\frac{1}{2} m-\frac{1}{4}\right), & \text { when } m \text { is odd. }\end{cases}
$$

Example 10 Similarly for any graph $H$ with $n$ vertices, using (11) the following result follows.

$$
\zeta\left(C_{m} \odot H\right)= \begin{cases}m(2 n+1)+\frac{1}{2} m^{2}(n+1), & \text { when } m \text { is even } \\ m(2 n+1)+\frac{1}{2} m(m-1)(n+1), & \text { when } m \text { is odd. }\end{cases}
$$

Example 11 One of the hydrogen suppressed molecular graph i.e., kenograph is bottleneck graph $(B)$ which is the corona product of $K_{2}$ and $G$, where $G$ is a given graph. So, by (11), total eccentricity of $(B)$ is given by $\zeta(B)=6 n+4$, where $n$ is the number of vertices of $G$.

The star graph $S_{n}$ on $n$ vertices is the corona product of $K_{1}$ and $\bar{K}_{n-1}$. The bistar $B_{n, n}$ is the graph obtained by joining the centre vertices of two copies of $K_{1, n}$ by an edge and is given by $P_{2} \odot \bar{K}_{n}$. So from the above formula the total eccentricities of these graphs are obtained as below.

Example $12 \zeta\left(K_{1} \odot \bar{K}_{n-1}\right)=2 n-1$ and $\zeta\left(P_{2} \odot \bar{K}_{n}\right)=6 n+4$.

Let $G$ be the graph of order $n$. Then the following result follows.

Corollary 5 The total eccentricity index of suspension graph $K_{1} \odot G$ is $\zeta\left(K_{1} \odot G\right)=2 n+1$.

The sunlet graph with $2 n$ vertices is obtained by attaching $n$ pendent edges to the cycle $C_{n}$. So it can be obtained by corona product of $C_{n}$ and $K_{1}$.

Example 13 For the sunlet graph $C_{n} \odot K_{1}$, we have

$$
\zeta\left(C_{n} \odot K_{1}\right)= \begin{cases}3 n+n^{2}, & \text { when } n \text { is even } \\ 3 n+n(n-1), & \text { when } n \text { is odd. }\end{cases}
$$

Let $G_{1}, G_{2}, \ldots, G_{n}$ be a set of finite pairwise disjoint graphs. The bridge graph with respect to the vertices $v_{1}, v_{2}, \ldots, v_{n}$, denoted by $B\left(G_{1}, G_{2}, \ldots, G_{n} ; v_{1}, v_{2}, \ldots, v_{n}\right)$ is the graph obtained by connecting the vertices $v_{i}$ and $v_{i+1}$ of $G_{i}$ and $G_{i+1}$ by an edge for all $i=$ $1,2, \ldots,(n-1)$. If $G_{1} \cong G_{2} \cong \ldots \cong G_{n}$ and $v_{1}=v_{2}=\ldots=v_{n}=v$, we define $G_{n}(G, v)=$ $B(G, G, \ldots, G ; v, v, \ldots, v)$. In particular, let $B_{n}=G_{n}\left(P_{3}, v\right)$ and $T_{n, k}=G_{n}\left(C_{k}, u\right)$ are special brige graphs. Then from definition of corona product of graphs, $B_{n} \cong P_{n} \odot \bar{K}_{2}$ and $T_{n, 3} \cong P_{n} \odot K_{2}$. 


\section{Example 14}

$$
\zeta\left(B_{n}\right)=\zeta\left(T_{n, 3}\right)= \begin{cases}\frac{9}{4} n^{2}+\frac{7}{2} n, & \text { when } n \text { is even } \\ \frac{9}{4} n^{2}+\frac{7}{2} n-\frac{3}{4}, & \text { when } n \text { is odd }\end{cases}
$$

\section{References}

1. Arezoomand, M., Taeri, B.: Applications of generalized hierarchical product of graphs in computing the Szeged index of chemical graphs. MATCH Commun. Math. Comput. Chem. 64(3), 591-602 (2010)

2. Arezoomand, M., Taeri, B.: Zagreb indices of the generalized hierarchical product of graphs. MATCH Commun. Math. Comput. Chem. 69(1), 131-140 (2013)

3. Barrière, L., Comellas, F., Dalfó, C., Fiol, M.A.: The hierarchical product of graphs. Discret. Appl. Math. 157, 36-48 (2009)

4. Barrière, L., Dalfó, C., Fiol, M.A., Mitjana, M.: The generalized hierarchical product of graphs. Discret. Math. 309, 3871-3881 (2009)

5. Dankelmann, M.O.P., Goddard, W., Swart, C.S.: The average eccentricity of a graph and its subgraphs. Util. Math. 65, 41-51 (2004)

6. De, N.: On eccentric connectivity index and polynomial of thorn graph. Appl. Math. 3, 931-934 (2012)

7. De, N.: Augmented eccentric connectivity index of some thorn graphs. Int. J. Appl. Math. Res. 1(4), 671-680 (2012)

8. Došlić, T., Graovac, A., Ori, O.: Eccentric connectivity index of hexagonal belts and chains. MATCH Commun. Math. Comput. Chem. 65, 745-752 (2011)

9. Eliasi, M., Taeri, B.: Four new sums of graphs and their Wiener indices. Discret. Appl. Math. 157, 794-803 (2009)

10. Eliasi, M., Iranmanesh, A.: Hosoya polynomial of hierarchical product of graphs. MATCH Commun. Math. Comput. Chem. 69(1), 111-119 (2013)

11. Eskender, B., Vumar, E.: Eccentric connectivity index and eccentric distance sum of some graph operations. Trans. Comb. 2(1), 103-111 (2013)

12. Fathalikhani, K., Faramarzi, H., Yousefi-Azari, H.: Total eccentricity of some graph operations. Electron. Notes Discret. Math. 45, 125-131 (2014)

13. Gutman, I.: Distance in thorny graph. Publ. Inst. Math. (Beograd) 63, 31-36 (1998)

14. Li, S., Wang, G.: Vertex PI indices of four sums of graphs. Discret. Appl. Math. 159, 1601-1607 (2011)

15. Luo, Z., Wu, J.: Zagreb eccentricity indices of the generalized hierarchical product graphs and their applications. J. Appl. Math., 2014 (2014), doi:10.1155/2014/241712

16. Metsidik, M., Zhang, W., Duan, F.: Hyper and reverse Wiener indices of F-sums of graphs. Discret. Appl. Math. 158, 1433-1440 (2010)

17. Tang, Y., Zhou, B.: On average eccentricity. MATCH Commun. Math. Comput. Chem. 67, 405-423 (2012) 